\title{
Troubles psychiques révélant une hypothyroïdie profonde
}

\section{Psychotic Disorders Caused by a Deep Hypothyroidism}

\author{
S. Köhl $\cdot$ M. Lutchmaya $\cdot$ P. Ray \\ Reçu le 8 novembre 2018; accepté le 28 janvier 2019 \\ (C) SFMU et Lavoisier SAS 2019
}

\section{Introduction}

Les manifestations psychiatriques font partie du tableau clinique de l'hypothyroïdie et sont classiquement caractérisées par un syndrome anxieux et/ou dépressif. Elles sont associées à des troubles somatiques qui dominent la scène clinique et conduisent à suspecter le diagnostic. Les troubles psychotiques au cours de l'hypothyroïdie sont connus depuis de nombreuses années $[1,2]$ mais rarement décrits. Ils apparaissent parfois au premier plan et rendent alors le diagnostic difficile. Le cas clinique rapporté est celui d'une personne prise en charge aux urgences pour un état délirant aigu révélant une dysthyroïdie profonde.

\section{Cas clinique}

Une femme de 56 ans, sans antécédent psychiatrique connu, célibataire et sans enfant, est adressée aux urgences par un médecin pour une hospitalisation sans consentement du fait d'un état délirant aigu. Elle est accompagnée par sa sœur qui rapporte une rupture de l'état antérieur avec repli sur soi et des troubles qui évoluent depuis plus de dix mois. L'interrogatoire du tiers retrouve un arrêt de l'activité professionnelle (elle est auxiliaire de vie) puis l'apparition récente de propos délirants sans notion de consultation ou de diagnostic psychiatrique récent. À l'arrivée dans le service, la patiente

\section{S. Köhl $(\bowtie) \cdot$ P. Ray $(\bowtie)$}

Centre régional universitaire des urgences, CHU François-Mitterrand,

5, boulevard Jeanne-d'Arc, F-21000 Dijon, France

e-mail : ko.sven@gmail.com, patrick.ray@chu-dijon.fr

M. Lutchmaya $(\bowtie)$

Service de psychiatrie adulte, CHU François-Mitterrand,

5, boulevard Jeanne-d'Arc, F-21000 Dijon, France

e-mail : marion_lutchmaya@hotmail.fr

P. Ray

Université de Bourgogne, 7, boulevard Jeanne-d'Arc, F-21000 Dijon, France présente une agitation importante nécessitant son installation en salle d'apaisement. L'infirmière organisatrice de l'accueil relève une pression artérielle à 136/96 $\mathrm{mmHg}$, une fréquence cardiaque à 65 battements/minute et une saturation en air ambiant à $96 \%$. L'examen clinique du médecin urgentiste est refusé par la patiente. L'entretien psychiatrique retrouve une femme sthénique avec une bizarrerie du contact, sans signe de confusion mentale. Elle verbalise des propos délirants dont l'adhésion est totale, de mécanismes interprétatif et intuitif, dont la thématique est polymorphe, à type de filiation (« ma sœur n'est pas ma sœur, car nous sommes adoptées », «je suis fille unique, orpheline depuis l'âge de deux ans dans les suites d'un accident de voiture de mes parents au Qatar où ils sont morts brûlés vifs ») et de persécution («les pompiers m'ont cassé une jambe », "Véronique (une connaissance) m'a faite interner, car je ne lui ai pas rendu un livre »). La thymie est neutre. Il n'est pas retrouvé d'élément en faveur de mécanisme hallucinatoire, et la patiente ne présente pas de désorganisation de la pensée.

Le seul antécédent médical connu et évoqué par le tiers est une épilepsie dans l'enfance, secondaire à de multiples traumatismes crâniens, traitée par phénobarbital et arrêtée il y a plus de 20 ans. L'analyse du dossier médical informatisé révèle une hypothyroïdie avec anticorps antithyroperoxydases (anti-TPO) positifs, diagnostiquée il y a six ans suite à un bilan biologique (réalisé en laboratoire de ville, et dont les valeurs ne sont pas mentionnées) lors d'une consultation dermatologique. La patiente n'a pas été supplémentée en hormones thyroïdiennes, car elle avait refusé la consultation en endocrinologie et avait été perdue de vue.

Le bilan biologique prélevé aux urgences ne montre pas d'anomalie ionique ou d'insuffisance rénale. Compte tenu des antécédents et de l'évaluation psychiatrique, un dosage immunologique par chimioluminescence (système Dimension Vista 1500) de TSH est réalisé avec un résultat élevé à $106 \mathrm{mUI} / \mathrm{ml}$. La FT4 est abaissée à 6 pmol/l. Un scanner cérébral est réalisé et ne montre pas d'anomalie.

Les troubles psychotiques aigus de cette patiente de 56 ans sans antécédent psychiatrique connu ont alors été attribués à l'hypothyroïdie profonde justifiant une orientation dans le 
service d'endocrinologie où la supplémentation en hormone thyroïdienne par lévothyroxine a été initiée à la posologie de $50 \mu \mathrm{g} / \mathrm{j}$ pendant trois jours puis augmentée à $75 \mu \mathrm{g} / \mathrm{j}$. Un traitement antipsychotique atypique par rispéridone instauré à $2 \mathrm{mg} / \mathrm{j}$ et augmenté progressivement jusqu'à $6 \mathrm{mg} / \mathrm{j}$ a été associé du fait de la sévérité du tableau psychiatrique. L'examen clinique réalisé secondairement au cours de l'hospitalisation a mis en évidence un goitre irrégulier et ferme ainsi qu'une peau pâle et infiltrée. La patiente a décrit une prise de poids rapide sans réussir à la dater ni la chiffrer. Il n’y avait pas de notion de constipation, d'hypothermie/frilosité ou de somnolence excessive. L'échographie thyroïdienne a confirmé la présence du goitre (de structure hypoéchogène et hétérogène), et le dosage des anticorps anti-TPO était élevé à $243 \mathrm{UI} / \mathrm{ml}$, permettant alors le diagnostic de thyroïdite de Hashimoto. L'évolution a été favorable avec une régression des signes physiques et une normalisation progressive du bilan thyroïdien (TSH à 3,78 mUI/l et FT4 à $17 \mathrm{pmol} / \mathrm{l}$ deux mois plus tard). Un traitement antipsychotique retard par palipéridone à la posologie de $150 \mathrm{mg}$ par mois (en relais de la rispéridone et pour des raisons pratiques) a néanmoins été proposé du fait de la persistance des symptômes psychotiques.

\section{Discussion}

La survenue d'un délire chez une personne âgée de plus de 50 ans sans antécédent psychiatrique connu est rare. Les principaux tableaux psychiatriques à évoquer sur ce terrain après avoir recherché et éliminé un syndrome confusionnel et/ou des hallucinations visuelles (particulièrement évocateur d'une cause organique) sont une psychose hallucinatoire chronique (schizophrénie à début tardif dans les classifications internationales), un trouble de l'humeur avec caractéristiques psychotiques et un délire paranoïaque. Ces diagnostics sont peu probables dans notre cas devant l'absence d'hallucination et de désorganisation de la pensée, une thymie neutre et le délire polymorphe. Les symptômes psychiatriques de la patiente peuvent être considérés comme l'expression d'une hypothyroïdie sévère.

Initialement décrite dans la littérature comme apparaissant bien après les signes physiques [3], il est démontré aujourd'hui que la symptomatologie psychiatrique de l'hypothyroïdie ne varie pas en fonction de sa profondeur [4]. L'hypothyroïdie peut s'exprimer par un tableau dépressif associant un ralentissement du débit verbal, une diminution des performances intellectuelles, une fatigabilité, une diminution de l'appétit et une apathie. La « folie myxœdémateuse », décrite pour la première fois en 1949 [1], réalise un tableau d'état psychotique confusodélirant et hallucinatoire ou d'état mélancolique fréquemment stuporeux, plus rarement d'hypomanie [5]. Cette dernière forme est rarement observée et peu décrite dans la littérature, ce qui souligne l'originalité de notre observation. Une étude transversale portant sur 45 patients suivis pour une hypothyroïdie périphérique a retrouvé un trouble psychotique pour seulement $2,8 \%$ des cas [6]. Lorsque les troubles psychotiques sont au premier plan, nous n'avons pas retrouvé d'étude comparant leur fréquence selon qu'ils sont en lien avec une hypo- ou une hyperthyroïdie. Le nombre de cas rapportés décrivant une dysthyroïdie révélée par une psychose est très faible et ne permet pas de déterminer une association privilégiée. Bien qu'établi [7], le lien entre ces troubles et la dysthyroïdie est encore mal compris. Plusieurs études évoquent l'action synergique des amines biogènes et des hormones thyroïdiennes impliquées dans de nombreux processus métaboliques qui auraient un rôle important dans la détermination de l'état psychiatrique [8].

En cas de forme neuropsychiatrique prédominante, l'hypothyroïdie peut être évoquée devant des signes cliniques qu'il convient de rechercher. Ils sont représentés essentiellement par un syndrome d'hypométabolisme (asthénie, somnolence, hypothermie, frilosité, constipation acquise, prise de poids pouvant contraster avec une anorexie), une atteinte cutanée et des phanères (peau pâle et sèche, dépilation, cheveux cassants) et un myxœdème cutanéomuqueux. L'agitation et le refus initial de l'examen clinique de l'urgentiste par la patiente n'avaient pas permis de les mettre en évidence lors de l'admission, mais certains de ces signes (prise de poids, peau pâle et infiltrée) ont été retrouvés secondairement au cours de l'hospitalisation. Dans le service d'accueil des urgences, c'est l'analyse du dossier médical, le terrain et l'entretien psychiatrique qui ont orienté vers une cause organique.

Une fois le diagnostic confirmé par la biologie (TSH augmentée, FT4 abaissée), le traitement hormonal substitutif permet habituellement la régression des signes physiques et l'amélioration des signes psychiatriques, en particulier s'il est initié précocement. En présence de symptômes délirants, un traitement psychotrope est associé à la supplémentation en hormones thyroïdiennes. Il est démontré que cette association permet une régression plus rapide des troubles psychotiques [9]. Aussi celui-ci est parfois imposé en cas de mise en danger par le patient du fait de comportements inadaptés provoqués par le délire. On choisira préférentiellement les antipsychotiques atypiques, en raison de leur meilleure tolérance et de leur plus faible risque de dyskinésies tardives qu'ils entraînent au long cours (par exemple rispéridone : $2 \mathrm{mg} / \mathrm{j}$, olanzapine : $7,5-10 \mathrm{mg} / \mathrm{j}$ ou aripiprazole : $5-10 \mathrm{mg} / \mathrm{j}$ ). L'olanzapine semble un traitement de choix du fait de son moindre risque sur le plan cardiologique [9-11], mais expose à une prise de poids importante. L'aripiprazole pourrait aussi être privilégié pour la même raison $[11,12]$, mais nous n'avons pas retrouvé d'étude où il était associé à la lévothyroxine pour le traitement de troubles psychotiques en lien avec une hypothyroïdie. L'arrêt des traitements psychotropes pourra être envisagé une fois le bilan thyroïdien stabilisé et les symptômes psychiatriques 
contrôlés. Ces symptômes persistent bien souvent plus longtemps que les troubles somatiques [9]. Aussi, il est estimé dans la littérature que $10 \%$ des patients présentent des symptômes neuropsychiatriques résiduels $[5,13,14]$, justifiant alors une prescription d'antipsychotique au long cours.

En conclusion, cette observation rappelle qu'une symptomatologie délirante chez une personne de plus de 50 ans sans antécédent psychiatrique connu peut être le témoin d'une pathologie organique notamment thyroïdienne imposant la recherche de signes cliniques évocateurs et la réalisation d'un dosage de la TSH.

Conflit d'intérêt : les auteurs déclarent ne pas avoir de lien d'intérêt.

\section{Références}

1. Asher R (1949) Myxoedematous madness. BMJ 2:555-62

2. Heinrich TW, Grahm G (2003) Hypothyroidism presenting as psychosis: myxedema madness revisited. Prim Care Companion J Clin Psychiatry 5:260-6

3. Logothetis J (1963) Psychotic behavior as the initial indicator of adult myxedema. J Nerv Ment Dis 6:561-8
4. Lehrmann JA, Jain S (2002) Myxedema psychosis with grade II hypothyroidism. Gen Hosp Psychiatry 24:275-7

5. Aarab C, Hammani Z, Aalouane R, et al (2016) Psychose aiguë secondaire à une dysthyroïdie : à propos de 2 cas. PAMJ 25:216

6. Belhadjsalah W, Chatti S, Mhalla A, et al (2014) Comorbidité psychiatrique de 1'hypothyroïdie. Ann Endocrinol (Paris) 75:510

7. Samuels MH (2014) Psychiatric and cognitive manifestations of hypothyroidism. Curr Opin Endocrinol Diabetes Obes 21:377-83

8. Constant EL, de Volder AG, Ivanoiu A, et al (2001) Cerebral blood flow and glucose metabolism in hypothyroidism: a positron emission tomography study. J Clin Endocrinol Metab 86:3864-70

9. Hynicka LM (2015) Myxedema madness: a case for short-term antipsychotics? Ann Pharmacother 49:607-8

10. Shlykov MA, Rath S, Badger A, Winder GS (2016) 'Myxoedema madness' with Capgras syndrome and catatonic features responsive to combination olanzapine and levothyroxine. BMJ Case Rep pii: bcr2016215957

11. Beach SR, Celano CM, Noseworthy PA, et al (2013) QTc prolongation, torsades de pointes, and psychotropic medications. Psychosomatics 54:1-13.

12. Aronow WS, Shamliyan TA (2018) Effect of atipical antipsychotic drugs on QT interval in patients with mental disorders. Ann Transl Med 6:147

13. Duval F (2003) Endocrinologie et psychiatrie. Encycl Med Chir, Psychiatrie. Elsevier SAS Ed., Paris, 10:28

14. Shinichi U, Satoko T, Motoki F, et al (2015) Acute psychosis as an initial manifestation of hypothyroidism: a case report. J Med Case Rep 9:264 